\title{
The economic impact associated with cerebrovascular events related to non-valvular atrial fibrillation (NVAF) in Italy: the role of apixaban
}

\author{
Lorenzo Pradelli ${ }^{1}$, Mario Calandriello ${ }^{2}$, Roberto Di Virgilio ${ }^{3}$, Marco Bellone ${ }^{1}$, \\ Marco Tubaro ${ }^{4}$ \\ AdRes, Health Economics \& Outcome Research, Turin, Italy \\ 2 Bristol-Myers Squibb Italy, Rome, Italy \\ 3 Pfizer Italy, Rome, Italy \\ 4 ICCU, Cardiovascular Department, San Filippo Neri Hospital, Rome, Italy
}

The presented supplement of Farmeconomia. Health Economics and Therapeutic Pathways focuses on the economic impact associated with cerebrovascular events related to non-valvular atrial fibrillation (NVAF) from the Italian National Health System (NHS) perspective.

A brief look into the scientific literature provides an overview on the burden of AF in the Italian population. In 2010, it was estimated that about one million of Italian patients are living with AF [1] and about $70 \%$ of them is affected by NVAF [2]. The prevalence of NVAF increases with age: much more than $10 \%$ of NVAF patients are over 80 years. The number of affected patients is expected to increase in the next years [2].

$\mathrm{AF}$ is associated with stroke and systemic embolism, and increased mortality. The current preventive measures include Vitamin $\mathrm{K}$ antagonists and antiplatelet agents, and recently new oral anticoagulants (NOACs) joined them. NOACs are offering a new option at the disposal of physicians to better manage risk of stroke and systemic embolism in NVAF patients. Physicians have to take the appropriate therapeutic choice: taking into account the embolic and hemorrhagic patient risk, and the risk associated with the use of anticoagulant therapy.

The pharmacoeconomic analyses below have been carried out by adapting a decision tree/ Markov model simulating the clinical experience of NVAF patients according to their probability of incurring an ischemic event, or cardiovascular disease. The first paper reports on a cost/effectiveness analysis of the use of apixaban relative to the standard of care in the prevention of thromboembolic events in vitamin $\mathrm{K}$ antagonist suitable and unsuitable patients. The second presents a budget impact analysis that compares NVAF patients eligible for treatment with NOACs in two scenarios - with and without apixaban. Clinical comparative effectiveness parameters among NOACs originate from on adjusted indirect comparison (Bucher's method) using warfarin as common comparator.

We hope that this information will support informed decisions by clinicians and especially payers.

\section{REFERENCES}

1. Bollettino Ufficiale della Regione Veneto, 2010. Available at http://bur.regione.veneto.it/ BurvServices/pubblica/HomeConsultazione.aspx

2. European Heart Rhythm Association, European Association for Cardio-Thoracic Surgery, Camm AJ, et al. Guidelines for the management of atrial fibrillation: the Task Force for the Management of Atrial Fibrillation of the European Society of Cardiology (ESC). Eur Heart J 2010; 31: 2369-429; http://dx.doi.org/10.1093/eurheartj/ehq278

Corresponding author Lorenzo Pradelli

I.pradelli@adreshe.com

\section{Disclosure}

Supplement funded

by Bristol-Myers

Squibb and Pfizer 